\title{
PRODUCTS OF ABELIAN SUBGROUPS
}

\author{
BERNHARD AMBERG AND W. R. SCOTT ${ }^{1}$
}

Abstract. This paper deals with several problems concerning groups $G=A B$ which are the product of two Abelian subgroups $A$ and $B$ and gives partial solutions under suitable finiteness conditions.

\section{Notations.}

c $X=$ centralizer of the subgroup $X$ of the group $G$, $\mathfrak{n} X=$ normalizer of $X$ in $G$,

$X \circ Y=$ set of all $x \circ y=x^{-1} y^{-1} x y$ with $x$ in $X$ and $y$ in $Y$,

$X \subseteq Y=X$ is a subgroup of $Y$,

$X \subset Y=X$ is a proper subgroup of $G$,

$X_{G}=$ largest normal subgroup of $G$ contained in $X$,

factor $=$ epimorphic image of a subgroup,

$F C$-group = group in which every element has only finitely many conjugates,

group with normalizer condition = group in which every proper subgroup is different from its normalizer,

two subgroups $A$ and $B$ of $G$ are locally conjugate if for every finite subset $X$ of $A$ there exists an element $g$ in $G$ such that the conjugates of elements in $X$ under $g$ are in $B$,

accessible subgroup = subgroup that may be connected with $G$ by a well-ordered ascending (normal) series.

Let the group $G=A B \neq 1$ be the product of two Abelian subgroups $A$ and $B$. We are interested in the following two properties:

(I) There exists a nontrivial normal subgroup of $G$ contained in $A$ or $B$.

(II) There exists a proper normal subgroup of $G$ containing $A$ or $B$.

By a well-known result of Itô every such group $G=A B$ with Abelian subgroups $A$ and $B$ is metabelian, i.e., $G^{\prime \prime}=1$. Itô also showed that (I) and (II) hold if $G$ is finite (see [3]). Cohn proved that (I) holds if $A$ and $B$ are infinite cyclic (see [2]). But it still seems to be unknown whether (I) or (II) holds if $A$ and $B$ satisfy the maximum

Presented to the Society, August 27, 1969 under the title On a result of Schenkman on products of abelian groups; received by the editors May 26, 1969 and, in revised form, March 13, 1970.

AMS 1969 subject classifications. Primary 2040, 2052.

Key words and phrases. Metabelian group, $F C$-group, groups with maximum or minimum condition, minimax group, product.

1 The second author was partially supported by NSF grant GP-12028. 
condition, since a proof of this result in Schenkman [4] contains an error (see Scott [5] or the review of [4]). Recently Sesekin showed in [6] that (I) holds if $A$ or $B$ satisfies the minimum condition.

In the following we give a few other results in this direction. For example (I) holds if $A$ or $B$ is accessible or if $G$ is an $F C$-group or an epimorphic image of a linear torsion group (Theorem 2). Also conditions are given under which (I) implies (II) (Proposition 3 and Theorem 4). For example (II) holds if $A$ or $B$ satisfies the maximum condition and the other the minimum condition or if $A$ and $B$ are both cyclic. Finally it is shown that if one of the subgroups $A$ and $B$ satisfies the minimum condition and the other is a direct product of a group with minimum condition and a group with maximum condition, then $G=A B$ is an extension of a minimax group by a minimax group (Theorem 5 ).

The first lemma gives some information on groups that do not satisfy (I). The main argument was already used in Schenkman [4].

LEMMA 1. Let $G=A B$ be the product of two Abelian subgroups $A$ and $B$ and assume that there exists no nontrivial normal subgroup of $G$ in $A$ or $B$. Then the following conditions must hold:

(a) $A \cap B=A \cap G^{\prime}=B \cap G^{\prime}=1$.

(b) $A$ and $B$ are not locally conjugate.

(c) $A=\mathfrak{n} A$ and $B=\mathfrak{n} B$.

Proof. If $A \cap B \neq 1$, then it is a nontrivial normal subgroup of $G$ contained in $A$ or $B$. Thus $A \cap B=1$. It has been shown by Itô in [3] that also $A \cap G^{\prime}=B \cap G^{\prime}=1$. Thus (a) is proved.

Itô has shown that $G$ is metabelian. If $A$ and $B$ were locally conjugate, then some conjugate of an element $a \neq 1$ in $A$ must be contained in $B$. Thus $a^{a^{\prime} b}$ is in $B$ for some $a^{\prime}$ in $A$ and some $b$ in $B$. This implies that $a$ is in $B$ which contradicts $A \cap B=1$.

Suppose next that $A \subset \mathfrak{n} A$, and let $D=\mathfrak{n} A \cap G^{\prime}$. If $D=1$, then $\mathfrak{n} A \simeq G^{\prime} \mathfrak{n} A / G^{\prime}$ is Abelian. If $D \neq 1$, then $A \circ D \subseteq A \cap G^{\prime}=1$ by (a), so that $A D$ is Abelian. In either case $A \subset \mathrm{c} A$. If $B^{*}$ is the set of elements of $B$ centralizing $A, \mathfrak{c} A=A B^{*}$ and $1 \neq B^{*}$ is a proper subgroup of $B$. But then $\mathrm{c} B^{*}=G$, and $B^{*}$ is a nontrivial normal subgroup of $G$ contained in $B$. By this contradiction $A=\mathfrak{n} A$. In the same way it may be shown that $B=\mathfrak{n} B$.

Theorem 2. Let the group $G=A B \neq 1$ be the product of two Abelian subgroups $A$ and $B$. Then there exists a nontrivial normal subgroup of $G$ in $A$ or $B$ if at least one of the following conditions is satisfied:

(a) $A$ or $B$ is accessible in $G$. 
(b) $G$ is an FC-group.

(c) $G$ is an extension of a torsion group with normalizer condition by a finite group.

(d) $G$ is an epimorphic image of a linear torsion group.

Proof. If this theorem is not true, then the conditions of Lemma 1 must hold. By condition (c) of this lemma $A=\mathfrak{n} A$ and $B=\mathfrak{n} B$ must be Carter subgroups of $G$. If $A$ or $B$ is accessible, then it is different from its normalizer, so that (a) cannot hold. In the other three cases Carter subgroups must be conjugate or at least locally conjugate, which contradicts condition (b) of Lemma 1. The local conjugacy of the Carter subgroups in a solvable $F C$-group has been shown by Stonehewer [8, p. 159]. The conjugacy of the Carter subgroups in a solvable extension of a torsion group with normalizer condition by a finite group has been shown by Stonehewer [7, Theorem 2.5, p. 527]. The conjugacy of the Carter subgroups in an epimorphic image of a solvable linear torsion group has been shown by Wehrfritz [9, Theorem 4.1, p. 152].

The following proposition gives a condition under which a condition of type (I) implies a condition of type (II).

Proposition 3. If the group $G=A B$ is the product of its subgroups $A \neq G$ and $B \neq G$ with $A \cap B=1$, then there exists a proper normal subgroup of $G$ containing $A$ or $B$ if the following two properties are satisfied:

(1) $B$ satisfies the maximum condition.

(2) If $N$ is a normal subgroup of $G$ and $G / N=(A N / N)(B N / N)$ where $A N / N \neq 1$ and $B N / N \neq 1$, then there exists a nontrivial normal subgroup of $G / N$ contained in $A N / N$ or $B N / N$.

Proof. There exist proper normal subgroups of $G$ of the form $X Y$ where $X$ is a subgroup of $A$ and $Y$ is a subgroup of $B$, and among these there exists one $A_{0} B^{*}$ where $B^{*}$ is maximal, since $B$ satisfies the maximum condition. If $B^{*}=B$, then $A_{0} B^{*}=A_{0} B$ is the required proper normal subgroup of $G$ containing $B$. Thus we may assume that $B^{*} \subset B$. Application of the maximum principle yields the existence of a subgroup $A^{*}$ of $A$ maximal with the property that $A^{*} B^{*}$ is normal in $G$. Since $A \cap B=1, A^{*} B^{*} \subset A B=G$. If $A^{*}=A$, then $A^{*} B^{*}$ is the required proper normal subgroup of $G$ containing $A$. Thus we may assume that $A^{*} \subset A$. It follows that

$$
\begin{gathered}
G / A^{*} B^{*}=\left(A B^{*} / A^{*} B^{*}\right)\left(A^{*} B / A^{*} B^{*}\right), \\
A B^{*} / A^{*} B^{*} \neq 1, \quad A^{*} B / A^{*} B^{*} \neq 1 .
\end{gathered}
$$

By (2) there exists a normal subgroup $N$ of $G$ containing $A^{*} B^{*}$ 
properly such that $N / A^{*} B^{*} \neq 1$ is contained in $A B^{*} / A^{*} B^{*}$ or in $A^{*} B / A^{*} B^{*}$. If the former holds, then for $A^{* *}=N \cap A$ we have $A^{*} B^{*} \subset N=A^{* *} B^{*} \subseteq A B^{*} \subset A B=G$ and $A^{*} \subset A^{* *} \subseteq A$, by which the maximality of $A^{*}$ is contradicted. If the latter holds, then for $B^{* *}=N \cap B$ we have $A^{*} B^{*} \subset N=A^{*} B^{* *} \subseteq A^{*} B \subset A B=G$ and $B^{*} \subset B^{* *} \subseteq B$, a contradiction to the definition of $B^{*}$. From this the assertion follows.

Two Abelian subgroups $A$ and $B$ of a group are said to be finitely connected if the following holds:

If $A^{*}$ is a subgroup of $A$ and $B^{*}$ is a subgroup of $B$ and $A^{*}$ and $B^{*}$ are isomorphic, then $A^{*} \simeq B^{*}$ satisfies the minimum condition (see Sesekin [6]).

Theorem 4. Let the group $G=A B$ be the product of two Abelian subgroups $A \neq G$ and $B \neq G$. Then there exists a proper normal subgroup of $G$ containing $A$ or $B$ if at least one of the following conditions is satisfied:

(a) $A$ and $B$ are finitely connected and one of them satisfies the maximum condition.

(b) $A$ or $B$ is accessible and one of them satisfies the maximum condition.

(c) $G$ is an $F C$-group and $A$ or $B$ satisfies the maximum condition.

(d) $A$ and $B$ are cyclic.

Proof. Since $A B$ is a group, $A B=B A$ and we may assume

(1) $B$ satisfies the maximum condition.

Let $N$ be a normal subgroup of $G$. Then $G / N=(A N / N)(B N / N)$ and $A N / N$ and $B N / N$ are Abelian and $B N / N$ satisfies the maximum condition. It is easy to see that conditions (b), (c) and (d) are satisfied by $G / N, A N / N$ and $B N / N$ if they are satisfied by $G, A$ and $B$. Assume now that $A$ and $B$ are finitely connected. Then one of them is a torsion group. Let $A^{*}$ be a subgroup of $A N / N$ and $B^{*}$ be a subgroup of $B N / N$, such that $A^{*}$ is isomorphic to $B^{*}$. Since $A^{*} \simeq B^{*}$ is a factor of $A$ and $B$ it is likewise a torsion group. But then, as an Abelian torsion group with maximum condition $B^{*} \simeq A^{*}$ is finite. This shows that $A N / N$ and $B N / N$ are finitely connected. Thus the following holds:

(2) If $A$ and $B$ satisfy one of the conditions (a), (b), (c) or (d), then this condition is also satisfied by $A N / N$ and $B N / N$.

Since $G$ is the product of two Abelian subgroups $A$ and $B, A \cap B$ is normal in $G$. We are ready now to apply Proposition 3 to $G / A \cap B$ $=(A / A \cap B)(B / A \cap B),(A / A \cap B) \cap(B / A \cap B)=1$. Clearly in all cases $B / A \cap B$ satisfies the maximum condition. If (b) or (c) is satisfied, then condition (2) of Proposition 3 follows from Theorem 
2. If (a) holds, then this condition follows from the theorem of Sesekin in [6]; and if (d) is valid it follows from Cohn's theorem if both the Abelian factors are infinite cyclic and again from Sesekin's theorem if one of them is finite cyclic. Application of Proposition 3 yields the existence of a proper normal subgroup of $G / A \cap B$ containing $A / A \cap B$ or $B / A \cap B$. It is clear then that there exists also a proper normal subgroup of $G$ containing $A$ or $B$.

Following R. Baer [1] an Abelian group $G$ is called a minimax group if it contains a normal subgroup $N$ satisfying the maximum condition such that $G / N$ satisfies the minimum condition. If $p$ is any set of primes, then the minimax group $G$ is a $p$-minimax group if $\mathfrak{p}$ contains each prime $p$ such that $G$ has an infinite $p$-factor group. A group $G$ is a meta-p-minimax group if $G^{\prime}$ and $G / G^{\prime}$ are both Abelian p-minimax groups.

Theorem 5. Let $\mathfrak{p}$ be a set of primes. If the group $G=A B$ is the product of two Abelian subgroups $A$ and $B$ of which one is a $\mathfrak{p}$-group with minimum condition and the other is the direct product of a group with maximum condition and a p-group with minimum condition, then $G$ is a meta-p-minimax group.

Proof. Since $A B=G=B A$ we may assume that the second factor $B$ is a $p$-group with minimum condition. If there exist groups $G$ satisfying the hypotheses of the theorem that are not meta-p-minimax groups we look at those where the torsion-free rank of $A$ is minimal.

Recall that any Abelian group $X$ with minimum condition is the direct product of finitely many groups $C_{p^{\infty}}$ of Prüfer's type for finitely many primes $p$ and a finite reduced part $F$. Let $c(X)$ be the number of factors of Prüfer's type occurring in such a decomposition and let $f(X)$ be the order of $F$. If $G=A B$ where $A$ is the direct product of a group with maximum condition and a $\mathfrak{p}$-group with minimum condition and $B$ is a $p$-group with minimum condition, let $r(G)$ be the sum of $c(\mathrm{t} A), f(\mathrm{t} A), c(B)$ and $f(B)$, where $\mathrm{t} A$ denotes the torsion subgroup of $A$. Among the groups with minimal torsion-free rank of the first factor that contradict the theorem we choose one $G=A B$ with minimal $r(G)$.

Let the subgroup $S$ of $G$ be an extension of a meta-p-minimax group by a meta-p-minimax group. Then by R. Baer $[1$, p. 27, Folgerung 4.4*], $S$ and all its factors are poly-p-minimax groups. By Itô's theorem $S$ is metabelian and since Abelian extensions of $p$-minimax groups by $\mathfrak{p}$-minimax groups are by $\mathrm{R}$. Baer $[1, \mathrm{p}$. 6] $\mathfrak{p}$-minimax groups, all Abelian factors of $S$ are p-minimax groups. We have shown:

(1) If the subgroup $S$ of $G$ is an extension of a meta-p-minimax 
group by a meta-p-minimax group, then $S$ is a meta-p-minimax group.

(2) $A_{G}=1$, and especially $A \cap B=1$.

By Sesekin's theorem there exist normal subgroups, not 1 , of $G$ contained in $B$. Thus $B_{G} \neq 1$, and $B_{G}$ satisfies the minimum condition as a subgroup of $B$. If $B_{G}$ were infinite, then $r\left(G / B_{G}\right)<r(G)$, so that $G / B_{G}$ is a meta-p-minimax group. But then by (1) $G$ is also a meta-pminimax group, a contradiction. Thus $B_{G}$ is finite. The following holds:

$$
G / B_{G}=\left(A B_{G}\right)\left(B / B_{G}\right) \text { and }\left(B / B_{G}\right)_{\left(G / B_{G}\right)}=1 \text {. }
$$

By Sesekin's theorem there exists a normal subgroup $N$ of $G$ such that $B_{G} \subset N \subseteq A B_{G}$. If $A^{*}=N \cap A$, then $A^{*} B_{G}=(N \cap A) B_{G}=N$. By (2) $A^{*}$ is not normal in $G$. Since $N$ is an extension of the finite Abelian $\mathfrak{p}$-group $B_{G}$ by the $\mathfrak{p}$-minimax group $A^{*} B_{G} / B_{G} \simeq A^{*}, N$ is a meta-pminimax group by (1). If $N$ contains elements of infinite order, then $G / N$ is a counterexample whose first factor $A N / N$ has torsion-free rank less than the torsion-free rank of the first factor $A$ of $G$. By this contradiction $N$ is a torsion group. If $N$ were infinite, then $r(G / N)$ $<r(G)$ and $G / N$ would be a meta-p-minimax group by the minimality of $r(G)$. It follows that $N$ must be finite. Then $G / \mathfrak{c} N$ which is essentially the group of automorphisms induced in $N$ by $G$ is also finite. But $A^{*} \subseteq N$ and $\mathrm{c} N \subseteq \mathrm{c} A^{*}$ and thus also $\mathrm{c} A^{*}$ has finite index in $G$. If $B^{*}$ is the set of elements of $B$ centralizing $A^{*}$, then $B^{*}$ is a subgroup and $\mathrm{c} A^{*}=A B^{*}$. Since $A^{*}$ is not normal in $G$ by (2), the index $\left|A B: \mathrm{c} A^{*}\right|=\left|A B: A B^{*}\right|=\left|B: B^{*}\right|$ is positive. But then $r\left(A B^{*}\right)$ $<r(A B)=r(G)$, so that $A B^{*}$ is a meta-p-minimax group. Since the index of $A B^{*}$ in $G$ is finite, $A B^{*}$ possesses only finitely many conjugates in $G$ and by the theorem of Poincare $G / A B_{G}^{*}$ is finite and therefore a meta-p-minimax group. But then by (1) $G$ is a meta-pminimax group, so that by this contradiction the theorem is proved.

Corollary 6. Let $G=A B$ be the product of two Abelian subgroups $A$ and $B$.

(a) If one of $A$ and $B$ satisfies the minimum condition and the other is the direct product of a group with minimum condition and a group with maximum condition, then $G$ is a metaminimax group.

(b) If $\mathfrak{p}$ is a set of primes and $A$ and $B$ are $\mathfrak{p}$-groups with minimum condition, then $G$ is a $\mathfrak{p}$-group with minimum condition.

Proof. (a) follows at once from Theorem 5 if $\mathfrak{p}$ is the set of all primes. If $A$ and $B$ are $\mathfrak{p}$-groups with minimum condition, then by Theorem $5 G$ is a meta-p-minimax group. Since $A$ and $B$ are torsion groups, it is an easy consequence of Sesekin's theorem that also $G$ is a 
torsion group. By R. Baer [1, p. 7, Folgerung 2.2], $G$ contains an Abelian normal $\mathfrak{p}$-subgroup $N$ with finite $G / N$. Then $G / N=(A N / N)$ $\cdot(B N / N)$ is the product of two finite $\mathfrak{p}$-subgroups and therefore a $\mathfrak{p}$ group. It follows that $G$ is a $\mathfrak{p}$-group with minimum condition.

\section{REFERENCES}

1. R. Baer, Polyminimaxgruppen, Math. Ann. 175 (1968), 1-43. MR 36 \#6501.

2. P. M. Cohn, A remark on the general product of two infinite cyclic groups, Arch. Math. 7 (1956), 94-99. MR 18, 376.

3. N. Ito, Über das Produkt von zwei abelschen Gruppen, Math. Z. 62 (1955), 400401. MR 17, 125.

4. E. Schenkman, The general product of two finitely generated abelian groups, Proc. Amer. Math. Soc. 21 (1969), 202-204; Zentralblatt Math. 174 (2), (1969), 308. MR 38 \#456.

5. W. R. Scott, On a result of Schenkman on products of abelian groups, Notices Amer. Math. Soc. 16 (1969), 796. Abstract \#667-137.

6. N. F. Sesekin, The product of finitely connected abelian groups, Sibirsk. Mat. Z. 9 (1968), 1427-1430=Siberian Math. J. 9 (1968), 1070-1072. MR 38 \#4559.

7. S. E. Stonehewer, Abnormal subgroups of a class of periodic locally soluble groups, Proc. London Math. Soc. (3) 14 (1964), 520-536. MR 29 \#2303.

8. - Locally soluble FC-groups, Arch. Math. 16 (1965), 158-177. MR 31 \#3511.

9. B. A. F. Wehrfritz, Soluble periodic linear groups, Proc. London Math. Soc. (3) 18 (1968), 141-157. MR 36 \#3894.

University of Texas, Austin, Texas 78712

University of Utah, Salt Lake City, Utah 84112 